\title{
Air pollution in the context of Chaotic Dynamics and Quantum Physics Paradigms
}

\author{
A. A. Berezin 1 , V. V. Gridin ${ }^{2}$ \& O. V. Yusim ${ }^{3}$ \\ ${ }^{I}$ Department of Engineering Physics, McMaster University, \\ Hamilton, Canada \\ ${ }^{2}$ Department of Chemistry, Technion-Israel Inst. of Technology, \\ Haifa, Israel \\ ${ }^{3}$ Scientific Research Institute for Atmospheric Air Protection \\ (SRI ATMOSPHERE), Saint-Petersburg, Russia
}

\begin{abstract}
Extending our previous papers (Air Pollution XIII, XVI and XVII), we discuss some physical aspects of Air Pollution (AP) and Global Air Pollution (GAP) stemming from the range of physical effects. We argue that these effects may produce a variety of chaotic and self-organizational consequences on GAP which affect the lateral dynamics and migratory mechanisms of GAP. Our prime attention is towards formation of collective states and collective excitations in AP/GAP system. We discuss same aspects of these effects in the context of chaos theory and quantum physics. We further discuss the potential purposeful structuring of electromagnetic perturbations for possible mitigation and passivation of AP effects on global and local scales.

Keywords: atmosphere, air chemistry, global air pollution, electromagnetic fields, cosmic factors, quantum collective effects, chaos theory, pollution transfer, pollution singularities, passivation of air pollution.
\end{abstract}

\section{Introduction}

In recent years significant progress was achieved in several key areas of human interest and activity by creative application of ideas and methods of physical sciences. While almost all areas of physics have useful applications, most important in recent period in terms of generating pivotal breakthroughs turned out to be Theory of Chaos, Complexity Theory and Quantum Physics. 
Theory of Chaos, or "Chaology" (which, technically, a sub-area of non-linear dynamics) provides radical insights into behavior and dynamics of interactive systems of varying complexity and scale. Such diverse areas as stock market, psychology and psychiatry, or global communication networks, came to the attention of chaos theorists. Complexity Theory, which, generically, has a significant overlap with Chaology, allows (using some ideas of physics) to approximate the behavior of complex systems with many degrees of freedom by relatively simple models with only a few degrees of freedom.

In this article we apply these ideas towards physics of air pollution (AP) and global air pollution (GAP). In-spite that there is no clear separation between AP and GAP (in fact, they are closely related), we use these terms provisionally, indicating that AP primary refers to local levels (communities, in-door air, etc), while GAP refers to a large scale patterns and dynamics. Our prime attention is towards formation of collective states and collective excitations in AP/GAP system. Examples of spontaneous formation of collective (macroscopic) coherent states abound at all scales, from nanometers (atomic scale) to megascale (terrestrial, and even cosmic scales). One of the insights which we owe to quantum physics and non-linear (chaotic) dynamics is the realization of the fact that small and week perturbations (seeds) can develop correlated collective states at large scale. Such universally known phenomena as Rainbow (light interference on water droplets), or Lightning (in which discharge channel propagates by a path of least electrical resistance), or Northern Lights (synchronous emission of ions in upper atmosphere) - all illustrate the fact that macroscopic systems can be highly connected and form collective states. In case of AP, as argued later in this article, some localized perturbations (singularities) can invoke collective states (patterns of AP/GAP) which sometime extend over hundreds and even thousands kilometers. In other words, Earth's atmosphere with its rich collection of charged and uncharged particles (ions, pollutant particulate, aerosols) turns itself to a "feast" of a large-scale collective behavior through charged and uncharged local singularities.

Another mighty example of large-scale correlated states is the formation of Tornados. The vertex of tornado is a huge singularity which behaves in a highly connected way. All these phenomena have aspects of disorder (e.g., percolation paths running by chains of random local singularities) and order (correlated collective dynamics).

Quantum physics, apart from its efficient mathematical methods, has also worked out an extensive library of terms and analogies. Some of them (like Uncertainty Principle, tunneling effect, quantum non-locality, etc) are powerful sources of further metaphors and conceptual analogies. This goes well beyond mere linguistic enrichments and often results in new heuristic tools. An example is the idea of Quantum Entanglement - a universal connectedness of quantum objects. But since, in-fact, all objects are "quantum" (classical physics is a limiting case of quantum physics), ideas of quantum entanglement are often transposed to macroscopic objects and systems. Later we consider the application of these ideas to the propagation of AP between distant regions. 
As in our previous papers on this topic [1-3], we propose our inferences as largely thought-provoking ideas to stimulate further theoretical research and practical solutions. Creative use of analogies, logical constructs and the very terminology taken from physical sciences, has a good track record in a broad range of areas from arts and humanities to engineering and economics [4]. In the area of air pollution science and technology, as in many other areas of human endeavor, the overabundance of facts and concrete data often makes a search of unifying concepts more difficult. Many facts are scattered, contradictory and poorly connected. Therefore, descriptive simplifications and generalizations offered by Complexity Theory often lead to useful insights and versatile models. In the same vein, one can take an analogy from arts - quite often a single sketch or a caricature made by just a few characteristic lines is capable to deliver more information and impression about a person or an event than "exact" photographs or detailed descriptions. That is why caricatures often have such a powerful impact in forming public perceptions ("memes"). This paper aims at proposing some new "memes" for air pollution science.

\section{Global air pollution}

In talking about AP or GAP, the first thing which comes into observation is a complex and interactive nature of AP/GAP. While at a certain level AP is seen as consisting of isolated pockets, it cannot be completely separated from other forms of pollution (water, soil, trash deposits, etc) or from such factors as electromagnetic fields or effects coming from outside the Earth (Solar activity, cosmic rays, etc). This results in a variety of descriptors typically used in AP/GAP discourse.

\subsection{Objective and subjective sides of air pollution}

As many other terms "air pollution" is not generically arise from any single conceptual image. It is not an "object" or an "effect" is the same pointed sense as these terms are traditionally understood in physics, and it cannot be measured or characterized in a singular way. Rather, it involves a whole gamut of descriptors at various spatial and temporal scales, some of these descriptors being localized, others are distributed.

\subsubsection{Objective characterization of air pollution}

In the most objective sense a complete characterization of GAP can be given - at least, in principle - by providing exact chemical and particulate composition in all points of space in time within Earth's atmosphere. By necessity all presently available models of weather prediction can use only a limited set of input parameters. As a result, the predictability of the existing quasi-deterministic computational algorithms often remains limited and non-uniform.

\subsubsection{Subjective aspects of air pollution}

Air pollution does not exist "as such". The character and level of air pollution is defined through the perception and criteria of human beings affected by it, 
whether they actually present in a particular area or could be. The subjective assessment of what is "clean air" and estimates of the level of cleanness/pollution may vary from person to person $[5,6]$. It also evolves with time and is subjected to regional, communal and professional variations. What is "clean air" for a city-dweller may not be so for someone living in high mountains. Same subjective variations may have even greater amplitude for the in-door air inside buildings or public transit. For example, in-door smoking in public places was almost universally unobjectionable just a few decades ago while presently it is prohibited in most business buildings and in almost all other public places.

\subsubsection{Structural descriptors of air pollution}

Almost any area of human activity and interests allows for a broad latitude of discourse and conceptual networking. In such areas as business, or city management and economics, we see activities ranging from day-to-day specific decisions or business deals to the most abstract mathematical models. In this vein AP/GAP can be naturally connected to a rich assortment of descriptive tools. Both fundamental mathematics (chaos, fractals) and concepts of quantum physics are among these tools. Such mathematical constructs as Fibonacci numbers, bifurcation cascades, Mandelbrot Set (etc) are used as heuristic models in many areas and some links to AP were discussed in by us earlier [1, 2]. Such methods as Simulated Neural Networks and Fuzzy Logic are actively used. In [7] we have discussed some of the related ideas in the context of the decision making for the preservation of heritage architecture. In fact, decision making in many different areas has the same kind of "self-similarity". That is a key reason why broad interdisciplinary education is so much emphasized for students in managerial and engineering areas.

\subsection{Air pollution as an open system}

Important aspect of AP and GAP is that in terms of physics they both constitute open systems. By that we mean that they cannot be totally isolated from outside interactions and influences. In quantum physics this often leads to such effects as loss of coherency in lasers, noise effects, etc. Similar effects for AP are mentioned below in this article.

\subsubsection{Air pollution as a subset of atmospheric physics}

Air quality, as represented by chemical composition and presence of hard and liquid particulate, is a natural characteristic of the atmosphere [1, 2]. Physics of global atmosphere is highly interactive. Vertical stratification and latitudinal motions of air masses (winds, vertices, etc) make it difficult to approximate the situation by two-dimensional models. In fact, the only truly adequate model is a 4-dimensional model of the detailed state of the atmosphere in space and time. They are mostly based on the use of diffusion equations which by necessity can describe the actual dynamics only approximately. 


\subsection{Physical factors in air pollution}

In a direct way AP is generated by chemical additives and micro particulate added to the air from various man-made and natural sources. As was mentioned above, what counts as AP is not always uniquely defined. For example, excessive levels of water vapor in the air (over-humidity) are normally not seen as AP, even if this affects breathing. Likewise, if air is overly dry (breathing difficulties at the other end), this situation as such does not qualify as AP either (unless, some other ingredients present). For in-door air either of these cases mitigated by the proper equipment - dehumidifies and humidifies, respectively. In this section we discuss other non-trivial contributing factors to AP which are of physical, rather than chemical, nature.

\subsubsection{Natural physical factors in air pollution}

It is well known that volcanic eruptions produce significant level of AP, sometime with long-term effects [1]. Likewise, black carbon generated by forest fires, coal power plants and vehicular exhausts injects micro and nano-soot to the atmosphere with major effects on air quality and radiative forcing. Likewise, there are other - less obvious - contributing factors to AP coming from magnetic and electrostatic fields and cosmic emissions (solar wind and high energy particles). None of these are contribution to AP directly; however their influence on AP is coming through a variety of physical effects stimulated by electromagnetic fields and outer (ex-planetary) emissions.

2.3.1.1 Magnetic fields can indirectly contribute to AP in a variety of ways. They affect motions of charged particulate through several physical effects which are mostly studied within the area of plasma physics [8-11]. Some of these effects were discussed in [2], here we add a few others which came to our attention recently. One useful analogy is a concept of exciton in solid state physics.

2.3.1.2 Exciton complexes can be formed in the presence of magnetic field through prior formation of Rydberg quantum states. Excitons are bound states of an electron and a hole and are formed in weekly bound atomic and molecular systems. Their energy spectrum reminds that of a hydrogen atom. Magnetic field affects the dynamics of excitons formation and diffusion, and can trigger energy transfer effects in dusty plasmas [8].

2.3.1.3 Electrostatic field effects have some well known AP aspects and some far less known. In general, static electric fields in the atmosphere are omnipresent phenomena due to natural charge separation. Clouds are often electrically charged. Effects of thunderstorm on the instantaneous local levels of AP are well known (like ozone formation). Usually, these effects are of a short duration and not very important in terms of AP as such. However, quantum approach to electrostatic field effects reveals more. One such plausible effect is a natural electrostatic precipitation due to induced dipole moments on microscopic 
particulate. This process resembles spontaneous distortion and charge redistribution in molecular complexes (Jahn-Teller effect). As a result, polarized particles have enhanced field-induced diffusion and can propagate faster and on greater distances.

\subsubsection{Civilization-generated factors in air pollution}

Along with natural perturbations, electromagnetic disturbances produced by human activity play ever-growing role in dynamics and patterns of AP [2]. There are several types of them, which are primarily either "power type" or "information type". The first are power generation facilities (nuclear and coal power stations, wind and solar photovoltaic generators, etc), as well as power transmission grids, residential power networks and numerous end-users (power consumers). At every stage these objects bear a role in local AP, sometime in indirect way by affecting the environment. The second type includes global communication networks, TV and broadcast, radio communications, including satellites as transmitters, etc. The informational disturbances have generally low power, but have greater degree of ordering and, hence, lower entropy. As a result of their ordered character, their influence on propagation of AP/GAP is often greater than that of power-based factors. The main effects in this case are coming through order-disorder transitions (order versus chaos) and resonance effect of several types, as discussed below.

2.3.2.1 Informationally structured electromagnetic emissions lead to numerous types of resonance interactions with chemical and mechanical nonuniformities in the atmosphere [2]. The first (chemical) refers to gaseous pollutants (e.g., car exhausts), the second (mechanical) indicates solid polluting particulate. Liquid particulate, such as aerosols, suspensions, and smog, fall inbetween [9-11].

2.3.2.2 Nanotechnology in the context of AP/GAP is another growing challenge. As more and more applied areas become entangled with nanotechnology (computers, informatics, drugs, genetics, new materials, etc), the problems related with the toxicity of nanoparticles are coming to the attention of researchers, engineers and public $[12,13]$. Such scenarios as "grey goo takeover" are becoming popular and, in-spite of their science-fiction flavor, they are not entirely free from more real concerns (e.g., presumed toxicity of self-replicating nanostructures).

\section{Non-linear and quantum effects}

Physical sciences were invariably at the frontier of technological progress. Furthermore, interaction between physics and technology is a two-way traffic, for example, in the course of the 19th century the development of steam engines (and later internal combustion engines) was closely related with progress in theoretical thermodynamics, such as the development of concept of entropy, etc. Likewise, the progress in electrical technology (both power and communication 
areas) was closely intertwined with advancements in theoretical electrodynamics (e.g., Maxwell's equations and wave theory).

At the present time (beginning of 21 st century), the prime emphasis has shifted towards environment, numerous aspects of a growing globalization of the world, as well as local pollution issues and health protection issues. Such novel areas of physical and mathematical sciences as Chaos Theory, Nonlinear Dynamics and Complexity Theory (all these are "umbrella" terms and each include many sub-fields) turn out to provide useful tools for many of the above mentioned social and environmental issues.

\subsection{Chaos theory in air pollution}

One of the central features of dynamical systems which are classified as chaotic is their extreme sensitivity to initial conditions [14] which is popular known as Butterfly effect. Thus, human-induced electromagnetic activities, even if they are energetically small, can produce significant alterations in patterns and behavior of AP/GAP. The possibility of fractal-like patterning of AP (similar to Mandelbrot Set) comes directly from non-linear nature of linkage between AP and atmospheric dynamics.

Complicated patterns of oscillations of Earth's magnetic field, as well as magnetic and electrostatic fields induced by power lines and communication networks, interact with the diffusional (unstructured) dynamics of AP propagation. This leads to a possibility that a chaotic behavior of AP can become (partially) structured with a net result that AP can propagate even to areas which were largely free from it in the regime dominated by a purely chaotic (unstructured) behavior. Examples of this are relatively high levels of AP and ground pollution deposition in some Arctic regions. Of specific concern is the precipitation of black carbon on ice which has an impact on planetary albedo and global radiation balance.

\subsubsection{Global communication networks}

Wireless global communication networks, largely based on satellites, transmit enormous volumes of highly structured information. In view that information in terms of statistical physics is a "negative entropy" (negentropy or syntropy), the effects of informationally-rich electromagnetic signals on the atmosphere and AP propagation can lead to resonance enhancement effects. An analogy of this effect is a forced crystallization in case when there are several different crystal symmetries for a particular chemical composition. A small crystalline seed (say, micro or even nano particle) of a particular crystal symmetry forces the entire volume attain the same symmetry upon crystallization.

\subsubsection{Singularity formations}

Non-linear systems are generally prone to the formation of singularities or other strongly localized dynamical features. An example is a formation of solitons in water channels or packs of localized phonons (lattice vibrations) in crystals. In terms of AP/GAP the formation of "singularities" (pockets of higher AP) may not necessarily be at the sites of major industrial centers (pollution emitters), but 
can be well outside them. In a non-linear diffusion physics an example of a similar process is DLA (diffusion limited aggregation) - a self-enhancing clustering of particles. These processes tend to interact both ways, for instance, AP-assisted clustering can chaotically disturb information-related global links.

\subsection{Quantum effects in air pollution}

Quantum physics provides a wealth of analogies and similarities for AP/GAP discourse. Experimental and theoretical developments in the area of the so-called macroscopic (large scale) quantum effects allows use of such concepts as effective mass or charge density waves to describe AP propagation. In solids conduction electrons, as a rule, have effective mass, m(eff), different from the free electron mass, $\mathrm{m}$ (free). This difference is due to imposition of periodical field of a crystal lattice which leads to the "renormalization" of electron mass. If (as it often happens in semiconductors), $\mathrm{m}$ (eff) $<\mathrm{m}$ (free), the electrons can move faster (have higher mobility) under an action of electric field. In AP case, similar effect (accelerated propagation) may come from a quasi-regular placement of major sources of pollution.

\subsubsection{Hopping propagation of air pollution}

Mechanism of hopping conductivity in semiconductors with impurities is an alternative to a "regular" drift-type electrical conduction. Hopping conductivity is based on quantum tunneling effect and can be additionally stimulated by external electric fields [15]. For propagation of AP/GAP the above mentioned ordering electromagnetic effects can lead to hopping percolation of AP streams and "quasi-quantum" tunneling to those places where AP was largely absent.

Another feature of hopping conductivity in doped semiconductors is the existence of "cul-de-sacs" - donor sites at which electrons can be trapped for a long time (such sites terminate percolation paths). In case of hopping propagation of AP this translates to a possibility that pockets of AP may "hang" for prolong time at some areas. The latter can be formed by some specific features of local landscape, like terrestrial depressions, mountain ridges or urban areas. In quantum analogy this is similar to the formation of localized bound states on a background of a continuous energy spectrum - so called NeumannWigner states, [17]. Likewise, for a small-scale AP situations (inside homes, cars, etc), some low-frequency low-intensity standing electromagnetic and/or acoustic waves can foster coagulation of micro-particulate into the nodes of such standing waves.

\subsubsection{Quantum entanglement}

The notion of quantum entanglement stems from non-local nature of quantum states (wave functions) as generally illustrated by Heisenberg Uncertainty Principle and EPR (Einstein, Podolsky, Rosen) gedanken experiment [16]. In some way, quantum non-locality and entanglement can be seen as an extension of Newton's "action-at-a-distance" ideas. In recent years similar notions were applied to some macroscopic (e.g., superconductors) and biological systems. In AP context quantum entanglement effects in aerosol particulate $[8,18]$ can lead 
to the formation of cohesive density waves which propagate by the mechanism of constructive interference, similar to the propagation of electromagnetic waves as described by Huygens principle (secondary wave re-emission). In this vein it is useful to recall an image of a Rainbow as a highly connected (and yet extended) result of light interference.

\subsubsection{Electrostatics and corona discharge effects}

Atmospheric electricity and discharge effects play a significant role in the dynamics of AP. Leaving aside ozone formation, other (more subtle) effects can lead to electrostatic phase transitions $[3,8,18]$ in AP pollution particulate with static charge. In the presence of corona discharge sources (e.g., high tension power lines) this can lead to electrohydrodynamic effects [11] and foster the formation of charge density waves and localized solitons.

\subsection{Patterning of global air pollution}

Due to various geographical, industrial and natural factors, the pattern of GAP is chaotic and unstable. However, as it is known from the Theory of Chaos, chaotic systems as a rule show patterns of order within chaos. These are due to various ordering factors.

\subsubsection{Ordering factors}

Numerous happenings of order within chaos range from micro to mega-scales. A well known example is Kirkwood gaps in the Asteroid Belt which are related to the gravitational ordering effect of Jupiter. In case of GAP and weather patterns, electromagnetic disturbances (natural and artificial), solar wind, seismic activity, etc, lead to a complicated and noisy frequency spectrum of perturbations. As a result, locally-predominant factors "take over" the Fourier decomposition of the spectrum and "collapse" it to a single (or a few) prime modes. This is similar to a reduction of the wave function in a quantum measurement process (popular illustration to that in quantum physics is a Schrodinger's Cat metaphor). This can lead to the formation of abnormal (anomalously strong) local islands of AP at the scale from several hundred meters to many kilometers. This illustrates a dichotomic nature of chaos which contains both disordering and ordering capacities.

\subsubsection{Inter-boundary and inter-continental pollution transfer}

For GAP at the continental scale (hundreds to thousand kilometers) the ordering aspects of chaotic dynamics may manifest themselves in a spontaneous fractalization of AP maps and the appearance of quasi-scale invariance (fluctuations in AP at different scales). Furthermore, the GAP system (as well as local AP subsystems) can show quasi-periodic temporal behavior (e.g., oscillations of different periods). Analysis of these effects on the basis of methods of classical and quantum chaos can potentially reveal new (so far undetected) fine features of AP dynamics and patterning. 


\section{Air pollution passivation}

The application of methods of Chaos Theory (such as using mass of data to calculate the Hausdorff dimension of embedded strange attractors) can shed a new light on possible novel methods of AP control. For example, targeted interference with AP (e.g., by high-energy probes such as electromagnetic pulses) at specific localities can trigger switching between different regimes of chaotic dynamics.

\subsection{Adaptation strategies}

Air pollution, as well as other forms of pollution, should not be seen as an absolute evil. Zero level pollution is a mere wishful thinking and a practical impossibility. Furthermore, in view of the hormesis effect [1] it is, actually, undesirable, even if it could be achieved. As a part of biosphere, humans evolutionary adapt to new forms of pollution, such as electromagnetic and informational [2], and new pollutants in AP (e.g., exhausts from nanotechnology).

\subsection{Air pollution passivation}

In view of the above mentioned need for a reasonable adaptation to AP, we can indicate several physics-based avenues for further feasibility studies as possible means of AP passivation. Pollution, like corrosion, cannot be totally eliminated, but both can be passivated to reasonably tolerable levels.

\subsubsection{Electrostatic screening}

Electrostatic screening is typically implemented by construction of Faraday cages. Application of a small electrical bias can lead to further effects. For example, similar to anodic passivation in metal corrosion (through the application of a voltage bias), AP can be passivated at a macroscopic scale by spatially isolating it from human habitat. Realistically, this can likely be applied for in-door pollution, inside vehicles, tunnels, etc.

\subsubsection{Quantum over-barrier reflection}

Macroscopic quantum effects have their analogies for AP particulate, like second hand smoking. Because most micro-particles of sub-micron size carry a small electrical charge (usually, one to a few elementary charges), creation of potential "electrostatic hills" by design of proper electrical biases (say, several volts) can deflect particles to desirable collection sites in an analogy with an operation of electrostatic precipitators $[3,11,19,20]$. This is a classical analogy of the effect of over-barrier reflection in quantum physics.

\section{Conclusion}

The main message of this paper is to suggest that AP/GAP can be productively studied as a quasi-quantum non-linear system with a high degree of internal 
connectivity as well as its inherent connectedness to other systems. The latter are such global entities as Earth's atmosphere with all its electromagnetic phenomena (natural and artificial), hydrosphere and terrestrial areas, as well as local pollution "singularities". We argue that in such highly connected and chaotic systems as AP/GAP one can expect the formation of collective excitations in analogy with other systems traditionally studied in modern physics.

As an issue of practical importance AP at all levels has many connections to human health [21] and as such needs to be treated in the context of global climate [22] and atmospheric processes [23]. While application of methods of traditional physics and chemistry to monitoring, control and modeling of AP is a massive area of activity, some new ideas can be imported from frontier areas of quantum physics and non-linear (chaotic) dynamics. Quantum and chaotic connections reviewed in this article by no means make a complete list of possible options for further explorations. Recent engineering applications of quantum non-localities (e.g., in information technology and quantum computing) and massive interdisciplinary applications of chaos theory are gradually making their way to the area of air pollution monitoring and control.

\section{References}

[1] Berezin, A.A. \& Gridin, V.V., Some aspects of air pollution in historical, philosophical and evolutionary context. Air Pollution XVII, ed. C.A. Brebbia \& V. Popov, WIT Press: Southampton, Boston, pp. 341-352, 2009.

[2] Berezin, A.A. \& Gridin, V.V., Electromagnetic and informational pollution as a co-challenge to air pollution. Air Pollution XVI, ed. C.A. Brebbia \& J.W.S. Longhurst, WIT Press: Southampton, Boston, pp. 533-542, 2008.

[3] Berezin, A.A., Quantum effects in electrostatic precipitation of aerosol and dust particles. Air Pollution XIII, ed. C.A. Brebbia, WIT Press: Southampton, Boston, pp. 509-518, 2005.

[4] Gregory, B., Inventing reality: physics as language. John Wiley \& Sons, Inc., New York, 1990.

[5] Jenkins, N., The general public's perception of air quality. Air Pollution VIII, ed. J.W.S. Longhurst, C.A. Brebbia \& H. Powel, WIT Press: Southampton, Boston, pp. 243-252, 2000.

[6] Kelay, T., Uzzell, D.L., Gatersleben, B., Hughes, S.J. \& Hellawell, E.E., Integrating scientific and lay accounts of air pollution. Air Pollution IX, ed. C.A. Brebbia \& J.W.S. Longhurst, WIT Press: Southampton, Boston, pp. 23-32, 2001.

[7] Berezin, A.A. \& Gridin, V.V., Complexity theory in management of heritage architecture preservation. STREMAH 2009 (Structural Studies and Maintenance of Heritage Architecture XI, ed. C.A. Brebbia, WIT Press, Southampton, Boston, pp. 255-266, 2009.

[8] Berezin, A.A., Quantum aspects of self-organization in dynamically random systems. Dusty and Dirty Plasmas, Noise, and Chaos in Space and 
in the Laboratory, ed. H. Kikuchi, Plenum Press, New York and London, pp. 225-240, 1994.

[9] Fews, A.P., Henshaw, D.L., Keitch, P.A., Close, J.J. \& Wilding, R.J., Increase exposure to pollutant aerosols under high voltage powerlines, International Journal of Radiation Biology, 75 (12), 1505-1521, 1999.

[10] Fews, A.P., Henshaw, Wilding, R.J. \& Keitch, P.A., Corona ions from powerlines and increased exposure to pollutant aerosols, International Journal of Radiation Biology, 75 (12), 1523-1531, 1999.

[11] Chun, Y.N., Chang, J.S., Berezin, A.A. \& Mizeraczyk, J., Numerical Modeling of Near Corona Wire Electrohydrodynamic Flow in a Wire-plate Electrostatic Precipitator, IEEE Transactions on Dielectrics and Electrical Insulation, 14 (1), 119 - 124, 2007.

[12] Ramsden, J.J. \& Freeman, J., The nanoscale, Nanotechnology Perceptions, $5(1), 3-25,2009$.

[13] Berezin, A.A., Stable isotopes in nanotechnology, Nanotechnology Perceptions, 5 (1), 27-36, 2009.

[14] Gleick, J., Chaos: Making a new science. Penguin Books, New York, 1988.

[15] Böttger, H. \& Bryksin, V.V., Hopping conduction in solids. AkademieVerlag, Berlin, 1985.

[16] Grib, A.A. \& Rodrigues, W.A., Nonlocality in quantum physics. Kluwer Academic/Plenum Publishers, New York, 1999.

[17] von Neumann, J. \& Wigner E.P., Uber merkwurdige diskrete Eigenwerte, Physikalische Zeitschrift, 30, pp. 465-467, 1929.

[18] Berezin, A.A., Coulomb correlation effects and pattern formation systems with multistabilities and breakdown, Journal of Electrostatics, 40\&41, 7984, 1997.

[19] Dela Cruz, E., Chang, J.S., Berezin, A.A., Ewing, D., Cotton, J.S. \& Bardeleben, M., Electrical effect of soot depositions in a co-axial wire pipe flow, Journal of Electrostatics, 67, 128-132, 2009.

[20] Chang, J.S., Tsubone, H., Chun, Y.N., Berezin, A.A. \& Urashima, K., Mechanism of electrohydrodynamically induced flow in a wire-non-parallel plate electrode type gas pump, Journal of Electrostatics, 67, 335-339, 2009.

[21] Holgate, S.T., Samet, J.M., Koren, H.S. \& Maynard, R.L. (eds), Air pollution and health. Academic Press, San Diego, 1999.

[22] Seinfeld, J.H. \& Pandis, S.N., Atmospheric chemistry and physics: from air pollution to climate change. John Wiley \& Sons, Inc., New York, 1998.

[23] Panofsky, H.A. \& Dutton, J.A., Atmospheric turbulence: models and methods for engineering applications. A Wiley-Interscience Publication, John Wiley \& Sons, New York, 1984. 